\title{
Reliable Packet Transmission over Multi-Hop Wireless Network
}

\author{
Prof. S.A.Bhuskute ${ }^{1}$, Ayesha Mulla ${ }^{2}$, Komal Mohite ${ }^{3}$ \\ Department of Computer Engineering, SCSCOE, Dhangawadi,Pune, India ${ }^{1,2,3}$
}

\begin{abstract}
In this paper we proposed A Fault node recovery algorithm to enhance the lifetime of a multi-hop wireless network when some of the sensor nodes shut down. The algorithm is based on the grade diffusion algorithm combined with the genetic algorithm. The algorithm can result in fewer replacements of sensor nodes and more reused routing paths.In networks, each sensor node has limited wireless computational power to process and transfer the live data to the base station or data collection center. Therefore, to increase the sensor area and the transmission area of the multihop wireless network usually contains many sensor nodes. Generally, each sensor node has a low level of battery power that cannot be replenished. Because of low battery power nodes are failure, the failed nodes will not relay data to the other nodes during transmission processing. Therefore using alternative path for reliable transmission of data to other node using trust value. A trust model is presented that allows the evaluation of the reliability of the routes.
\end{abstract}

Keyword: Genetic algorithm, grade diffusion (GD)algorithm, Fault node recovery algorithm, Trustvalue, Multi-Hop wireless networks(WN).

\section{INTRODUCTION}

In wireless networks, every sensor node haslimited Node Determination. The nodes will be replaced with new wireless computational power for processing and nodes of same nodeid this process is called Fault Node transferring the data to the base station or data collection Recovery. The FNR algorithm replaces the deactivated center. So that, to increase the sensor area or processing sensor nodes and uses more reused routing paths. area and the transmission area in which data transfer from one node other node, the wirelessnetwork have to many In [3], Author proposes a fault node recovery algorithm to sensor nodes. Generally, each sensor node has a low level enhance the lifetime of a wireless sensor network when of battery power so that it cannot be recovered. The failed some of the sensor nodesshut down. The algorithm is nodes which have not anymore power to transfer data. The based on the grade diffusion algorithm combined with the sensor node which failed to transfer data will notrelay data genetic algorithm. The algorithm can result in fewer to the other nodes during transmission processing. Thus, replacements of sensor nodes and more reused routing the other sensor nodes will be load with paths.

increasedtransmission processing. This paper proposes a fault node recovery (FNR) algorithm with trust valueto In [4], Author proposes the opportunistic routing paradigm increase the lifetime of a multi-hop wireless network is a new avenue for designing routing protocols in multi$(\mathrm{WN})$ and reliable transmission of data.At time when some hop wireless networks. In opportunistic routing protocols, of the sensor nodes shut down in transmission area \& they all neighboring nodes that are closer to the destination may do not have longer battery power (energy) or they have overhear a data packet, and may be a candidate to reached theiroperational threshold. Using the FNR forwarding the packet for its destination. In [5], Author algorithm we have result insome replacements of sensor proposes a fault node recovery algorithm with trust value nodes and more reused routingpaths. This, algorithm not to increase lifetime of wireless network and reliable only increases the wireless network (WN) lifetimebut also reduces the cost of replacing the sensor nodes.

\section{RELATED WORK}

\subsection{Literature Servay}

In [1],Author proposes a fault node recovery (FNR) algorithm to enhance the lifetime of a multi-hop wireless network (WN) when some of the sensor nodes shut down, either becausethey no longer have battery energy or they have reached their operational threshold. Using the FNR algorithm can result in fewer replacements of sensor nodes and more reused routing paths.

In [2], Author proposes process of finding the set of nodes whose battery power is less than threshold is called Fault When in wireless network some of the sensor node shut down because of low battery power, using replacement of nodes it can find alternative routing path, and using trust value for reliable transmission of data.

In [6], Author proposes an algorithm based on ladder diffusion and ACO is proposed to solve the powerconsumption and transmission routing problems in wireless networks. The proposed ladder diffusion algorithm isemployed to route paths for data relay and transmission inwireless sensor networks, reducing both power consumptionand processing time to build the routing table andsimultaneously avoiding the generation of circle routes. In [7], the proposed algorithm considers 
recovery of eachnode based on the distance of each node 3.2. Grade Diffusion Algorithm

from cluster heads,remained energy of cluster heads and The Grade Diffusion (GD) algorithm to improve the member number of each cluster head and selects the best ladder diffusion algorithm using ant colony optimization cluster head torecovery.

(LD-ACO) for wireless sensor networks. The GD In [8], a trustmodel is presented that allows the evaluation algorithm creates the routing path for each sensor node.It of thereliability of the routes, using only first- also identifies a set of neighbor nodes to reduce the handinformation.

\section{PROPOSED WORK}

\subsection{System Design:}

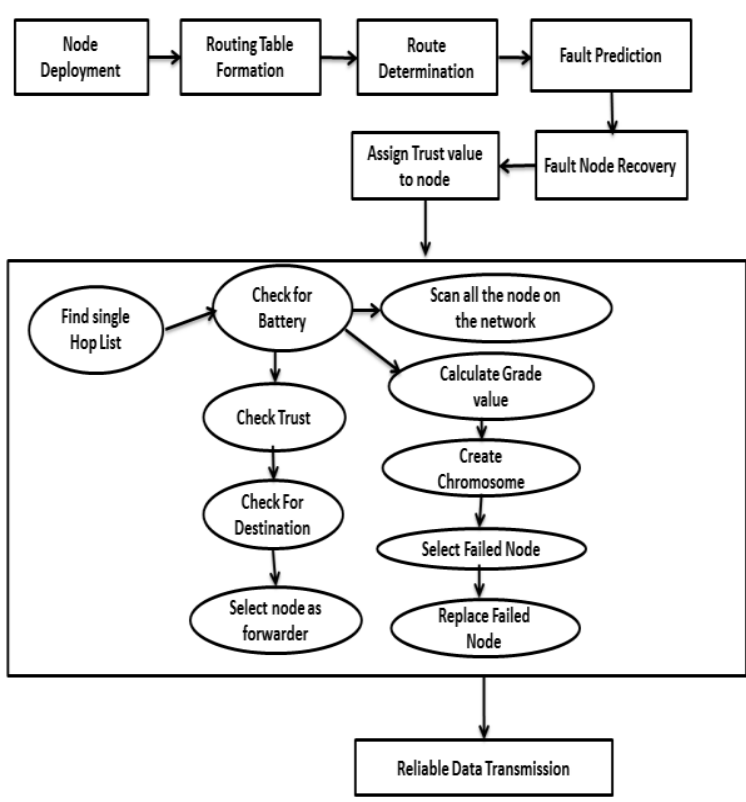

Fig.1.SystemDesign Architecture

\section{Node Deployment Algorithm-}

This algorithm is responsible for deployment of nodes in aparticular area. This will position the nodes in the given area

\section{Routing Table Formation-}

This is the algorithm which is used to form routing tables foreach of the nodes. The routing table will contain informationabout other nodes in the network in terms of node id anddistance of each node w.r.t other nodes in the network.

\section{Route Determination-}

This is the process which involves determining the routefrom the source node to destination node with the aid ofusing the control packets and the route must be found insuch a way that battery consumption is reduced and overallnetwork lifetime is also increased.

\section{Fault node Prediction-}

This is the process in which the node's whose battery poweris below than certain threshold is determined

\section{Fault Node Recovery-}

This is the process by which the nodes whose battery poweris below threshold are determined and replaced with the newnodes but with same node id. transmission of data.

$$
\begin{aligned}
& \text { Sensor Node } \\
& \text { Sink Node }
\end{aligned}
$$

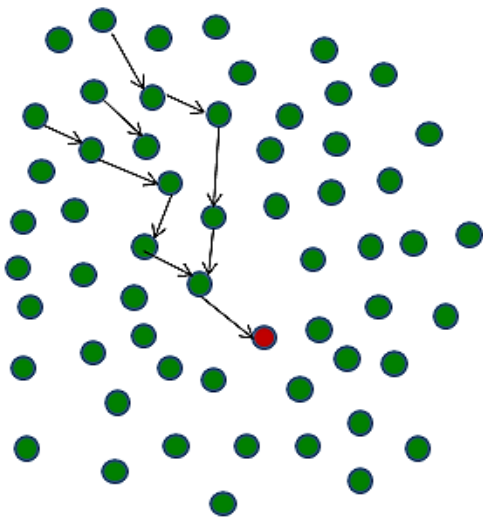

Fig. 2.Wireless sensor node routing

This algorithm providegrade table for each sensor node. When its grade table defect node able to perform the relay. Each sensor nodecan select a sensor node from the set of neighbor nodes. The GD algorithm can also record some information regarding the data transmission. Then, a sensor node can select nodes which have alighter loading or more available energy or power than the other nodes to perform the extra transmission operation. That means, the GD algorithm can updates the routing path inreal time, and the data is sent to the sink node quickly and correctly. Whether the DD or the GD algorithm is applied, the grade-creatingpackages or interested query packets must first bebroadcast to all sensor node. Then, the sensor nodes transfer the event data tothe sink node, according to the algorithm, when suitable eventsoccur. The sensor routing paths are shown in Fig. 3

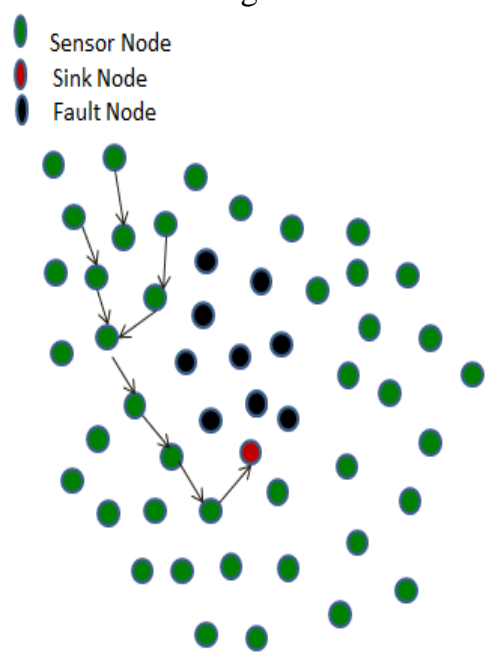

.Fig.3. Wireless sensor node routing path when some nodes are not functioning. 
The WSN may fail due to a variety of causes, includingthe following: the routing path might be break; theWSN sensing area might be leak; the batteries ofsome sensor nodes might be depleted, requiring more relaynodes; or the nodes damaged after the WSN has been inuse a long period of time. In Fig. 2, the situation in whichthe outside nodes transfer event data to the sink node viathe inside nodes (the sensor nodes near the sink node) in aWSN to provide the accommodation measures for nonworkingnodes. The inside nodes thus have the largest data transmissionloading, consuming energy at a faster rate. If all the inside nodes reduce their energy or otherwise stop to function, theevent data can no longer be sent to the sink node, and theWSN will no longer function. The power of the sensor nodes in WSNs isunavoidable. This paper, however, proposes an algorithm tosearch for and replace fewer sensor nodes and to reuse themost routing paths. Also using trustworthiness to transmite reliable data to sink node.

\subsection{Route Discovery Process}

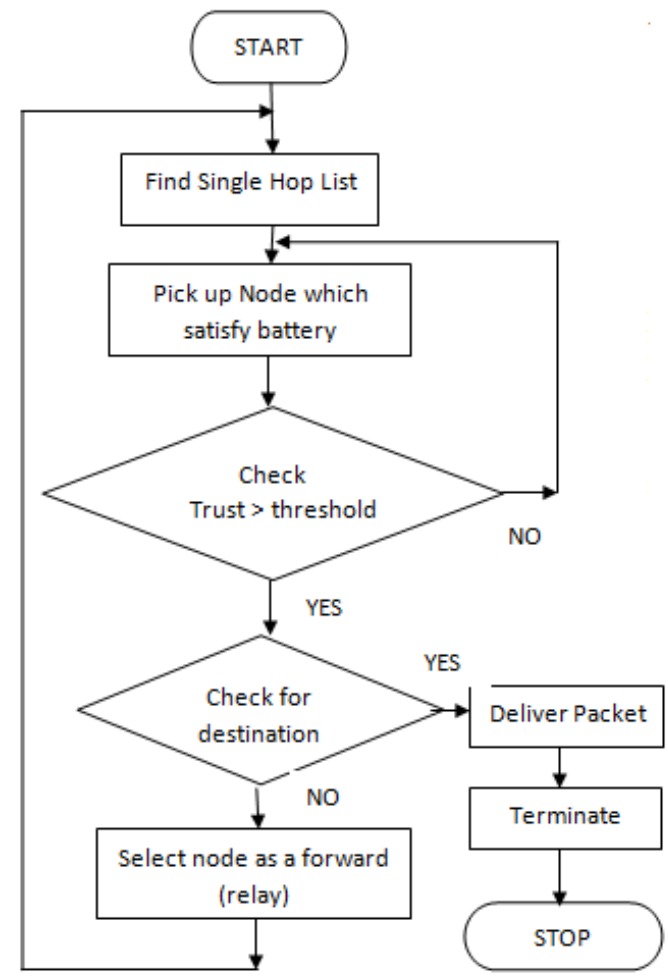

Fig. 4 shows the route discovery process using grade diffusion algorithm.

In the current approach, a route discovery approach is proposed which reduces amount of power consumption. The process of finding the set of nodes whose battery power is less than threshold is called Fault Node Determination. The nodes will be replaced with new nodes of same node id this process is called Fault Node Recovery.

Fault Node Detection and Recovery Algorithm to send packets from source node to destination node. The algorithm has following steps:
1) The sensor node maintains a single hop list, which contains the ids of all nodes within its transmission range.

2) When a source node wants to send control packet to the sink, it includes a packet threshold with initial value $\mathrm{N}$ in each control packet.

3) The REQ packet is flooded to the single hop list.

4) Each neighbor will then send the REQ packet by picking the nodes which has highest battery power.This process is repeated until the link is establishedtill the destination.

5) If packet threshold is zero then a process is followed by picking a node which helps us to reach the destination faster.

\subsection{Genetic Algorithm}

The Genetic algorithm is one of the best energy efficient algorithms in wireless sensor networks. It optimizes the signal strength of sensor nodes. This algorithm also helps in reducing the energy consumption and thus increases thelifetime of wireless sensor networks. This algorithm consists of five steps i.e., i) Initialization ii) Evaluation iii)Selection iv) Crossover v) Mutation.

\section{i) Initialization}

It is first step of the Genetic algorithm.In initialization step the genetic algorithm generate chromosomes. The total number of chromosomes will be the number of nonfunctioning nodes. The genes values will be 0 or 1 .

\section{ii. Evaluation}

The second step in Genetic algorithm is Evaluation. Here in this stage fitness function is evaluated by providingfitness values. The fitness function is defined over the genetic representation and measures the quality of therepresented solution. The fitness function is always problem dependent. The input parameters will be chromosomes itself.

$$
f_{n}=\sum_{i=1}^{\max (\text { Grade })} \frac{P_{i} \times T P^{-1}}{N_{i} \times T N^{-1}} \times i^{-1}
$$

Where,

$N i=$ the number of replaced sensor nodes and their gradevalue at $i$.

$P i=$ the number of re-usable routing paths from sensornodes with their grade value at $i$.

$T N=$ total number of sensor nodes in the original WSN

$T P=$ total number of routing paths in the original WSN

\section{iii. Selection}

The third step in Genetic algorithm is Selection. The main aim of this step is to select the chromosomes. Chromosomeshaving the highest fitness value. First it selects the pair of chromosomes from the node.The selected chromosomes which is having highest fitness value will be send to the mating pool to produce new set of chromosomes which will happen in the crossover step. 


\section{iv. Crossover}

The Crossover step in Genetic algorithm is used to create individual chromosomes. The two individual chromosomes will be selected from the mating pool to generate a new set of offspring. A one-point crossover is selected between the two parents and then the fraction of each of the individual according to the crossover will be swapped.

\section{v. Mutation}

The mutation step can introduce traits not found in the original individuals and prevents the GA from converging toofast. The sensor nodes will be replaced in the chromosomes with gene of 1 in order to get better network lifetime.

\subsection{Node failure detection:}

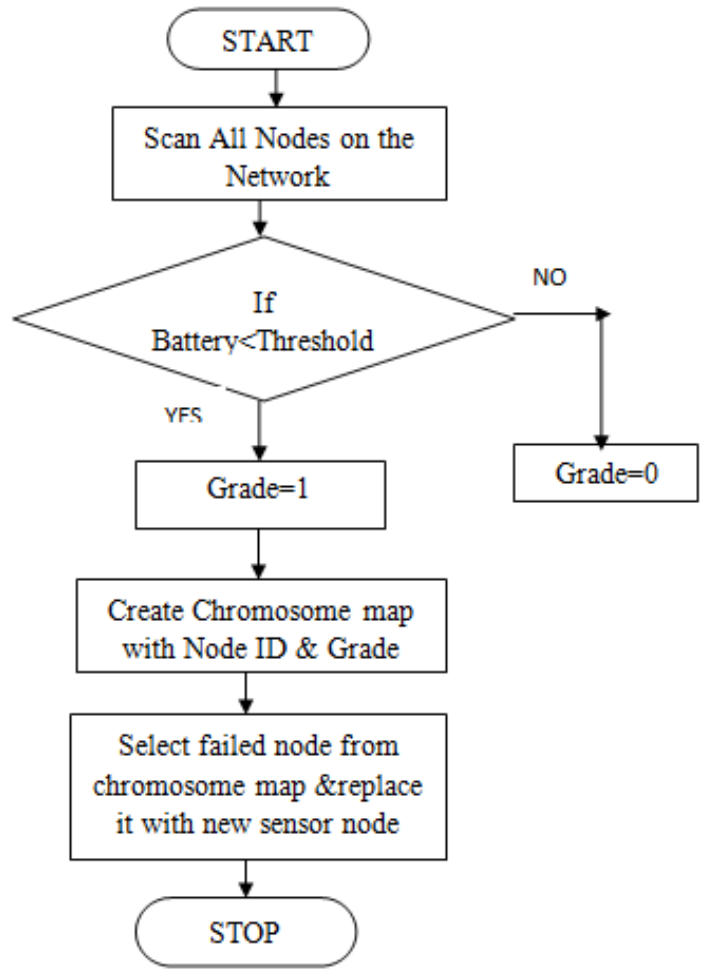

Fig 5: shows Node failure detection using Genetic Algorithm

The above flowchart shows the Node Failure detectionprocess:

1) The Nodes are scanned from the first node onwards and link is established to collect data from each single

2) hop list

3) The mutation is computed by comparing the battery power with the threshold.

4) Each node is assigned a grade value of 0 or 1 . A 1 is assigned if the battery power of node is less thanthreshold otherwise it will be assigned a value of 1.

5) A chromosome Map is created which will contain key as Node ID and value as the grade value.

6) Set of nodes is determined from the map which have a value as 1
6) The process is repeated until all nodes have been scanned.

\subsection{FAULT NODE RECOVERY ALGORITHM}

This paper proposes a fault node recovery (FNR) algorithm for wireless sensor network based on the grade diffusion algorithm combined with the genetic algorithm.

The FNR algorithm creates the grade value, routing table,neighbor nodes, and payload value for each sensor nodeusing the grade diffusion algorithm. In the FNR algorithm, the number of non-functioning sensor nodes is calculatedduring the wireless sensor network operation, and theparameter $\mathrm{B}_{t h}$ is calculated according to equation. The sensor nodes transfer the event data to the sink node according to the GD algorithm when events appear. Then, $\mathrm{B}_{t h}$ is calculated according to equation in the FNR algorithm. If $\mathrm{B}_{t h}$ is larger than zero, the algorithm will be invoked and replace nonfunctioning sensor nodes by functional nodes selected by the genetic algorithm. Then the wireless sensor network can continue to work as long as the operators are willing to replace sensors.

$$
\begin{gathered}
\mathrm{B}_{t h}=\sum_{i=1}^{\max (\text { grade })} \mathrm{T}_{i} \\
T_{i}=\left\{\begin{aligned}
1, & \frac{N_{i}^{\text {now }}}{N_{i}^{\text {original }}}<\beta \\
0, & \text { otherwise }
\end{aligned}\right.
\end{gathered}
$$

Where,

$N_{i}^{\text {original }}=$ is the number of sensor nodes with the Grade value $\mathrm{i}$

$N_{i}^{\text {now }}=$ is number of sensor nodes still functioning at the current time with grade value $\mathrm{i}$

$\beta=$ value between 0 and 1 .

\subsection{TRUSTOPPORTUNISTIC PROTOCOL:}

A trust model is presented that allows the evaluation of the reliability of the routes, using only first-hand information. Trust value of each node should be assigned and computed in advance. If a node joins the network or the network is firstly working, its trust value is set as a initial value 0.5

When network topology changes, the trust value of each node can be updated by formula (1) and (2), and moreover.

If trust value of a node is less than Tthreshold,this node will be elicited as a selfish or malicious node, so it will be omitted in next routing phrase from security of network point of view.

\subsubsection{Generate WSN Topology:-}

Let $\mathrm{G}=(\mathrm{V}, \mathrm{E})$ denote the topology of the network. Assume that node $\mathrm{j}$ is neighbor node $\mathrm{i}$. It is denoted by $T_{j}(i, n)$ trust on node $\mathrm{j}$ assigned by node i. $T_{j}(i, n)$ is $n^{\text {th }}$ topology computed by, 
$T_{j}(i, n)=\frac{F_{i j}(n)}{N_{i j}(n)}$

Where,

$N_{i j}(n)=$ the number of packets that have been received by $i$ at time $n$.

$F_{i j}(n)=$ the number of packets that have been received by $i$ at time $n$.

\subsubsection{Implementation GDA with Trust value:-}

Calculate trust value of node by using a moving average model. Using $(n+1)^{t h}$ topology

$T_{j}(i, n+1)=\alpha \cdot T_{j}(i, n)+(1-\alpha) \cdot T_{j}(i, n+1)$

Where,

$T_{j}(i, n+1)=$ is node $\mathrm{j}$ 's trust value measured by node $i$ during the $(n+1)^{\text {th }}$ topology updating cycle.

$\alpha=0<\alpha<1$ is a weighting factor used to tradeoff between current measurement and previous estimation.

\section{SIMULATION AND RESULT}

The simulation of fault node recovery algorithm described in the above section has been performed to verify the methods and results.

The grid based topology is used to place the nodes. It will work for any topology. All the nodes will be given with same battery energy at the initial state. The graph for this is also shown. After some iterations there will be a loss of energy when packets has been sent from source node to sink node.

The fault node recovery algorithm is more useful as and when more number of iterations is considered. After some iteration there will be energy loss, and then FNR algorithm will check for the threshold count and trust value.

Using alternative routing path transfer the packet reliably from source node to destination.To compare the performance of the proposed protocol the following metrics were considered:

1) Throughput refers to how much data can be transferred from one location to another in given amount of time.

2) Packet delivery ratio(pdr) is the ratio between the number of successfully received packets and the number of generated packets.

3) Packet loss ratio(plr) is the ratio between the numbers of packets loss.

4) Average end-to-end delay per packet is the end-toend delay (in seconds) for successfully received packets.

5) Normalized routing overhead is the number of routing packets per one data packet that is successfully receive at the destination.

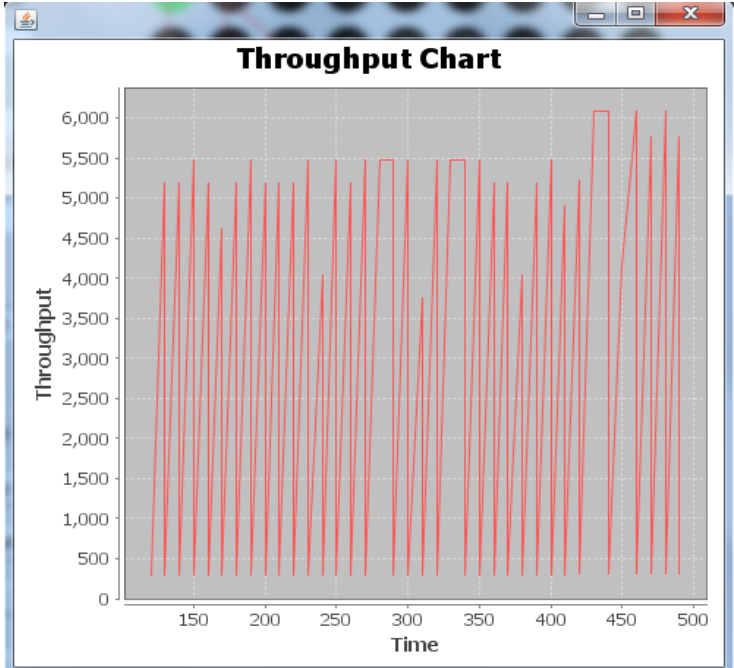

Fig.6 Time vs throughput

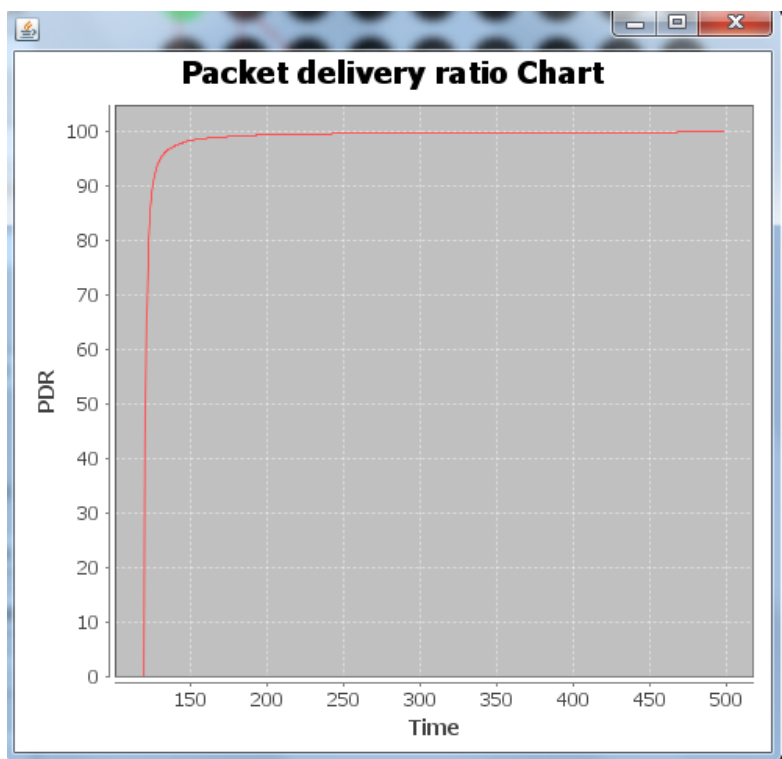

Fig.7 Time vs Packet delivery ratio

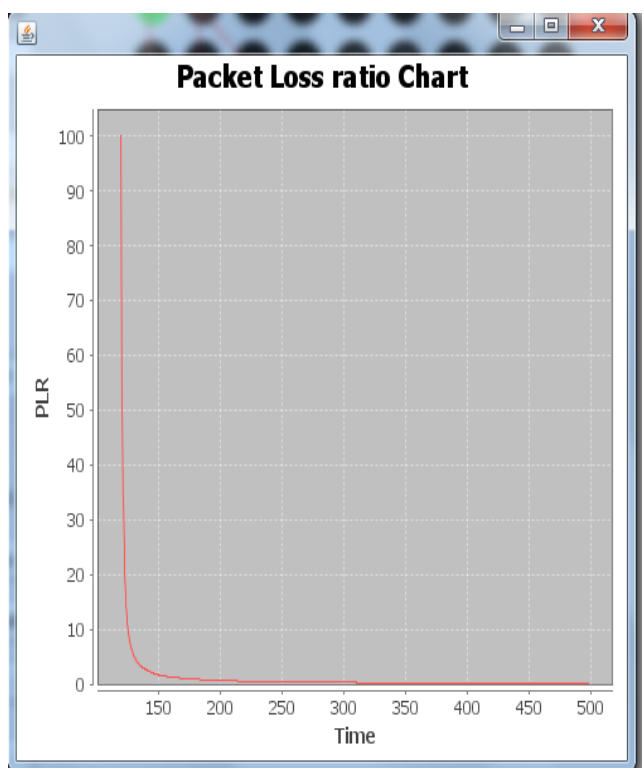

Fig.8 Time vs Packet loss ratio 


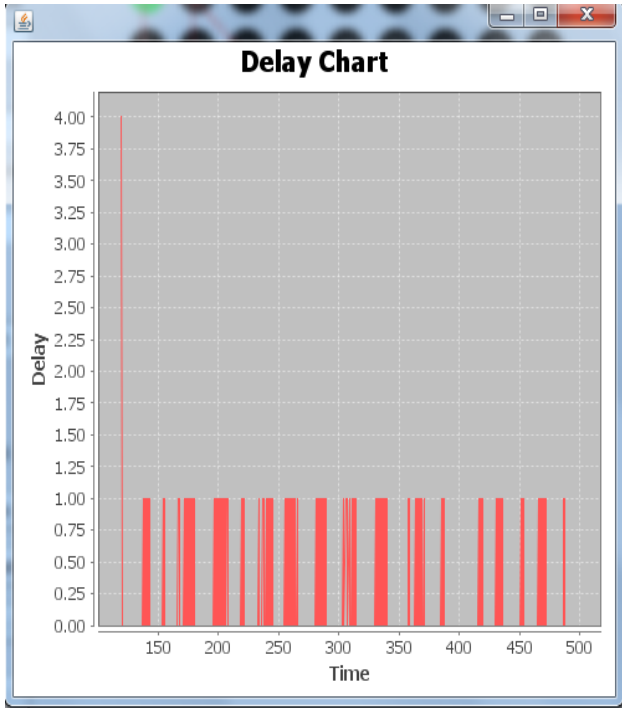

Fig.9 Time vs Delay

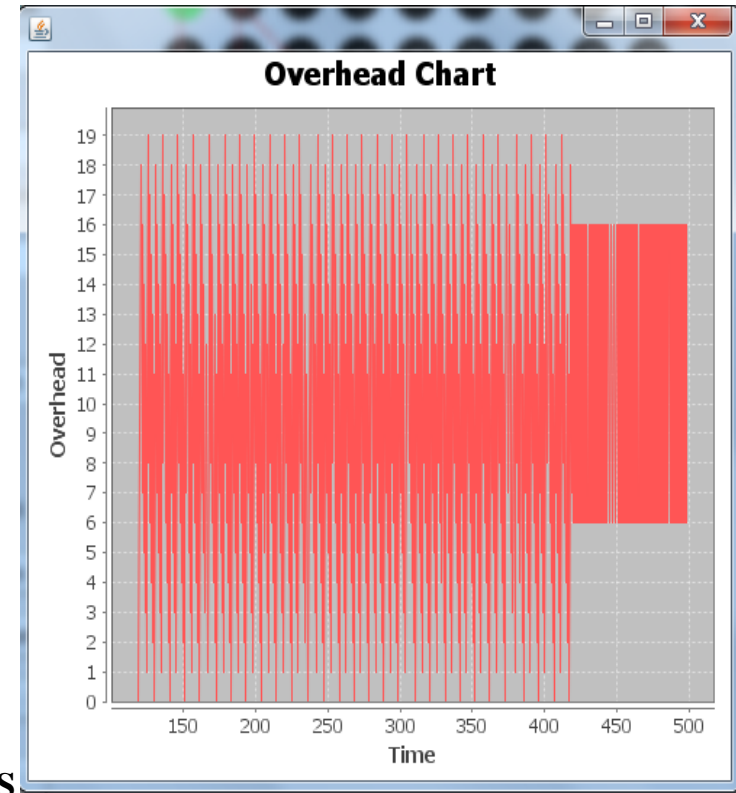

Fig.9 Time vs Overhead

\section{CONCLUSION}

In multi-hop wireless networks, the sensor nodes use battery power supplies and thus have limited energy resources. In addition to the routing, it is important to research the optimization of sensor node replacement, reducing the replacement cost, and reusing the most routing paths when some sensor nodes are non-functional. The various simulations demonstrate that the round trip time, Number of Hops. The FNR algorithm using Trust value requires replacing fewer sensor nodes and reuses the most routing paths, increasing the WSN lifetime and reducing the replacement cost and transmission of data is reliable.A trust model is presented that allows the evaluation of the reliability of the routes.

\section{REFERENCES}

1]. Hong-Chi Shih, Jiun-Huei Ho, Bin-Yih Liao, and Jeng-Shyang Pan, "Fault Node Recovery Algorithm for a Wireless Sensor Network," IEEE sensor journal vol.13 no. 7, 2013.
[2]. Pavithra B Raj, R. Srinivasan, "Fault Node Identification and Route Recovery in distributed Sensor Network," vol.3, May 2014.

[3]. Sharanapriya J,Rekha Patil,"Design and Implementation of Dead Nodes Recovery Algorithm to Improve the Lifetime of a Wireless Sensor Network," vol.3, August 2014.

[4]. Wango, Haung Chuanhe, Yang Wenzhong, WangTong, "Trust Opportunistic Routing Protocol in Wireless Network," 2010.

[5]. S.A.Bhuskute,Ayesha Mulla, Komal Mohite, Madhuri Mane, "Reliable Transmission with Fault Node Recovery Algorithm in Wireless Sensor Network," Int.jrnl.sci.vol.3, pp.2319-7064 Dec. 2014

[6]. J. H. Ho, H. C. Shih, B. Y. Liao, and S. C. Chu, "A ladder diffusion algorithm using ant colony optimization for wireless sensor networks, ’'Inf. Sci., vol. 192, pp. 204-212, Jun. 2012.

[7]. T. P. Hong and C. H. Wu, "An improved weighted clustering algorithm for determination of applicationnodes in heterogeneous sensor networks," J. Inf. Hiding

[8]. A.A.Pirzada, C.McDonald, Establishing trust in pure ad-hoc networks,in: Proceedings of the27th Australasian Computer ScienceConf.(ACSC'04),2004, pp.47 $\square 54$.

[9]. J. A. Carballido, I. Ponzoni, and N. B. Brignole, "CGD GA: A graph based genetic algorithm for sensor network design," Inf. Sci., vol.177, no. 22, pp. 5091-5102, 2007.

[10]. S. Corson and J. Macker, Mobile Ad Hoc Networking (MANET): Routing Protocol Performance Issues andEvaluation Considerations. New York, NY, USA: ACM,1999

[11]. J. H. Ho, H. C. Shih, B. Y. Liao, and J. S. Pan, "Grade diffusion algorithm," in Proc. 2nd Int. Conf. Eng. Technol. Innov., 2012, pp. 2064-2068

[12]. J. Pan, Y. Hou, L. Cai, Y. Shi, and X. Shen, "Topology control for wireless sensor networks," in Proc. 9th ACM Int. Conf. Mobile Comput.Netw. 2003, pp. 286-299. 